\title{
Cooling of High Power Generation Systems: Matching Demands with Supplies across the Length and Time Scales
}

\author{
Andrei Fedorov \\ Georgia Institute of Technology, Institute for Bioengineering and Bioscience \\ NSF ERC for Cell Manufacturing Technologies (CMaT) Love Bldg. \\ Room 303, 771 Ferst Drive Atlanta, Georgia 30332-0405 \\ AGF@gatech.edu
}

\begin{abstract}
I will start my talk by introducing the challenges of thermal management and unique aspects/needs for cooling technologies across the length scales from an individual transistor ( nanometers) to data centers and large-scale IT infrastructure (10s of meters). I will present a simple scaling analysis of various cooling methods in terms of their coefficient of performance (cooling capacity/density per unit power input required for operation) and cooling system size, which could be used by a system designer for matching the application demands with cooling capabilities. The second part of my presentation will illustrate different active and passive approaches for supercharged cooling, primarily of "hot spots" with ultra-high power density, using the single phase liquid and hybrid (single phase + two-phase) cooling approaches in 2D and 3D architectures. These include gas-assisted evaporation, microgap two-phase convective boiling of high pressure refrigerants, electrospray-assisted thin film evaporative cooling, and composite solid-to-liquid capacitors for fully passive cooling with off-duty regeneration. If time permits, I will also touch upon new ideas on use of nanostructures to facilitate high efficiency dropwise condensation via long-range droplet coalescence for condensate removal. The details on science and engineering design aspects of these cooling technologies have been broadly disseminated in archival literature and intellectual property is available for in depth consideration by interested parties.
\end{abstract}

